\title{
Introductory Text to Sonochemistry
}

\author{
Muthupandian Ashokkumar \\ School of Chemistry, University of Melbourne, VIC 3010, Australia \\ masho@unimelb.edu.au
}

Sonochemistry is a multi-disciplinary and an emerging research field that has potential applications in many areas that include chemistry, physics, biology, materials, food science, engineering, and medicine. The primary focus of this article is to provide a basic, textbook-type introduction highlighting the fundamental aspects and selected applications of sonochemistry. The purpose of this article is not to provide a full literature review, but to give the readers a birds' eye view of the basics of acoustic cavitation and selected examples of applied sonochemistry. Extensive review articles and book chapters are available that provide in depth analysis of current literature in various topics.

\section{Introduction}

Humans and animals use sound waves for communication. Sound passes through various media as sine waves of positive (compression) and negative (rarefaction) pressure cycles (Figure 1). The speed of sound is dependent on the nature of the medium through which it propagates. For example, the speed of sound is about $342 \mathrm{~m} / \mathrm{s}$ and $1440 \mathrm{~m} / \mathrm{s}$ in air and water, respectively.

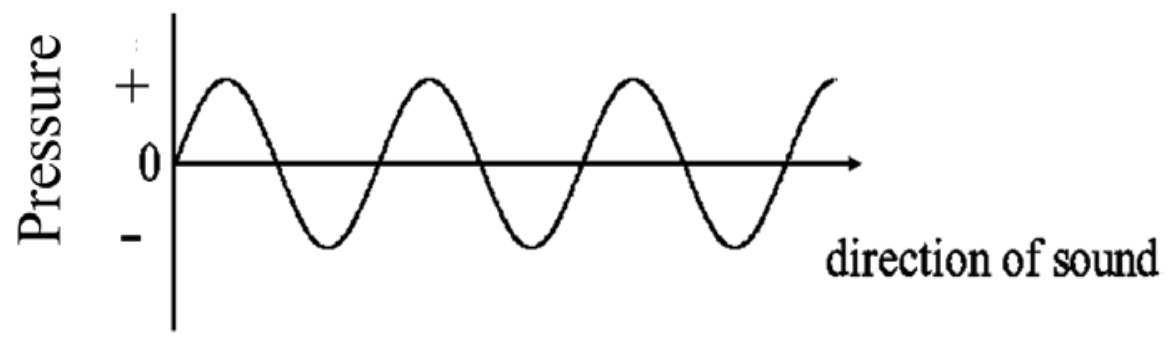

Figure 1: Propagation of sound waves.

Sound waves of $>20 \mathrm{kHz}$ (20000 Hz (cycles/s)) frequency are referred to as ultrasound since they are beyond human hearing range. In recent times, ultrasound has been found useful in a variety of applications [1-3]. In Figure 2, some key applications are highlighted. Numerous studies have investigated the use of ultrasound for synthesising organic, inorganic and biomaterials with unique properties. Ultrasonic processing has resulted in the modification of physical and functional properties of proteins, carbohydrates and other constituents of food and dairy systems. Other applications include wastewater treatment, sonocrystallisation, emulsification, extraction, deemulsification, etc. 


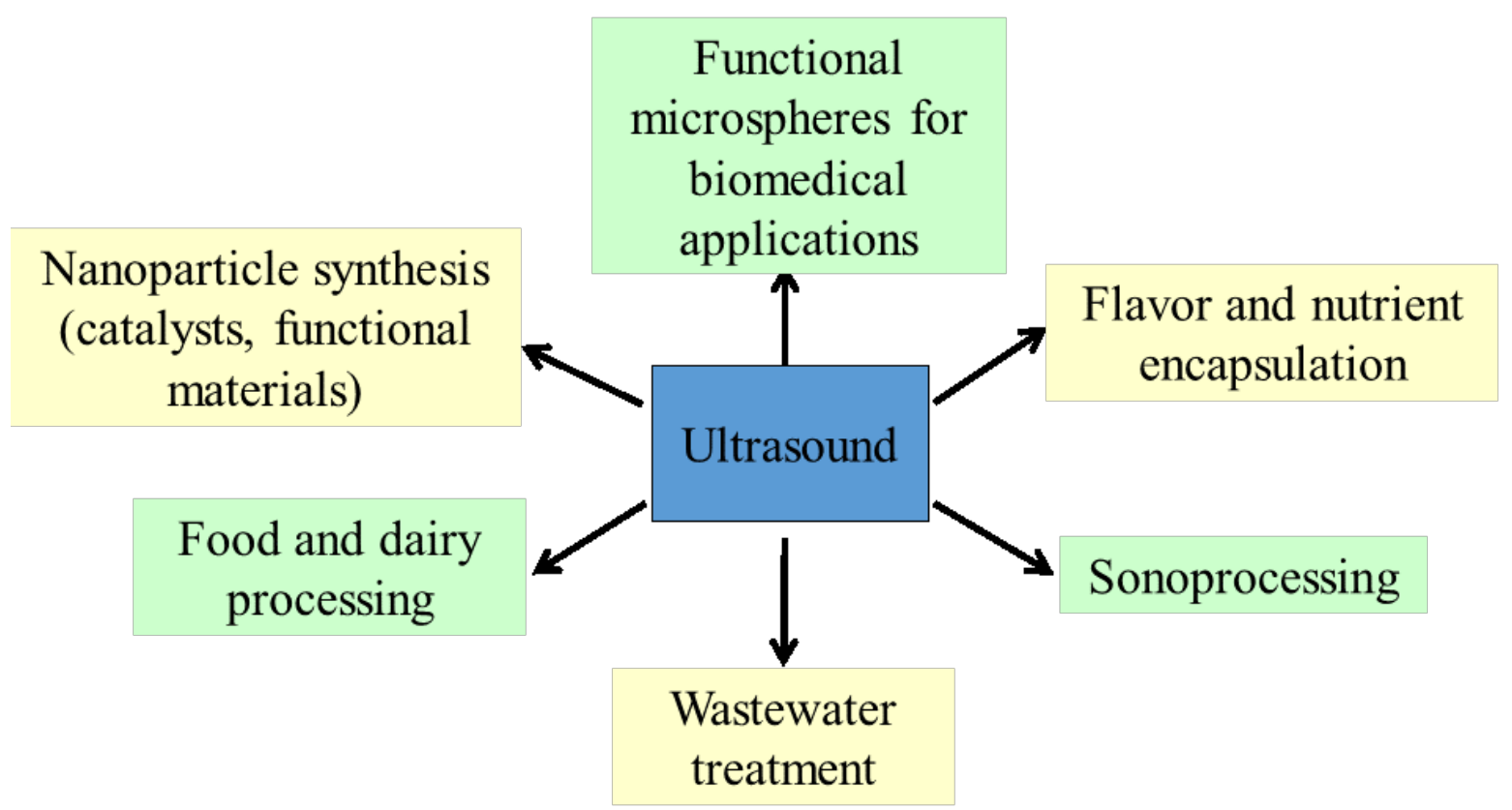

Figure 2: Selected applications of ultrasound. For further reading, References [1-3] provide several review papers on these applications.

Most of the applications arise due to a phenomenon called Acoustic Cavitation [4]. When sound waves pass through a medium, part of the acoustic energy is absorbed by the medium that results in heating of the medium. If the medium is a liquid, such as water, acoustic cavitation could occur depending upon the "strength" of sound waves and properties of the liquid.

\section{Acoustic cavitation}

“Cavitation” literally means creation of a cavity in a liquid medium. For example, if enough energy is provided, intermolecular forces could be overcome to generate a cavity or bubble. Such energy could be provided through hydrodynamic forces, heating or photons (focused laser beams). When acoustic force is used, it is referred to as acoustic cavitation. Using Equation 1 [4], the threshold negative pressure that is required to overcome the cohesive forces of a liquid to create a cavity of radius $\mathrm{R}$ could be calculated.

$$
\left.P_{\mathrm{B}} \approx P_{\mathrm{h}}+0.77 \sigma / R \quad \text { (Equation } 1\right)
$$

where $P_{\mathrm{B}}, P_{\mathrm{h}}, \sigma$ and $R$ represent threshold pressure, pressure in the liquid, surface tension of the liquid and radius of the cavity, respectively. Using $P_{\mathrm{h}}=1 \mathrm{~atm}(101,325 \mathrm{~Pa})$, surface tension of water $(0.072 \mathrm{~N} / \mathrm{m})$, a critical negative pressure of about 500 atm could be estimated to create a cavity/void/bubble of radius $0.1 \mathrm{~nm}$. 
However, such a high energy is not required to achieve cavitation under normal circumstances due to the existence of bubble nuclei in liquids. All liquids used in experiments under normal laboratory conditions would contain dissolved gas molecules that are also responsible for the existence of small gas pockets on the surface of the containers, in particular when there are some defects such as crevices. When such bubble nuclei exist, not much energy is required to create them.

As mentioned earlier, the literal meaning of cavitation is creation of a cavity. However, acoustic cavitation refers to the growth of existing bubble nuclei (or small gas pockets trapped in impurity particles or on the surface of containers, etc.) under the influence of acoustic force followed by their inertial collapse. The growth of bubble nuclei in an acoustic field occurs due to "Rectified Diffusion” process [5]. When bubble nuclei experience negative pressure of the sound waves, they are forced to expand during which dissolved gas and solvent molecules diffuse into the bubble. During the compression phase of the acoustic cycle, due to an increase in pressure inside the bubbles, gas molecules would diffuse out. Due to a difference in surface areas of expanded and compressed states of the bubble, and changes in concentration of gases in the liquid shell around the bubble (Ref. 5 provides detailed description of the area and shell effects), the amount of gas/vapor that diffuses into the bubble dominates over that diffuses out, resulting in a net growth of the bubble over many acoustic cycle.

The growth of bubbles under acoustic force is "controlled" by applied ultrasonic frequency. Equation 2 shows a simplified relationship between frequency and resonance radius of bubbles.

$$
F * R \sim 3 \quad \text { (Equation 2) }
$$

where, $F$ is ultrasonic frequency in ' $\mathrm{Hz}$ ' and $R$ is the resonance radius of the bubble in meters. As per Equation 2, bubbles below $R$ would grow until they reach $R$ for a specific applied frequency. Using Equation 2, the resonance size of bubbles at $20 \mathrm{kHz}$ would be about $150 \mu \mathrm{m}$ and that at 500 $\mathrm{kHz}$ would be $6 \mu \mathrm{m}$. It should also be noted that the experimentally measured resonance size of bubbles is much smaller than what is predicted using Equation 2 due to various experimental parameters, such as liquid viscosity, etc. For example, experimentally determined resonance radius of cavitation bubbles at $20 \mathrm{kHz}$ is about $5 \mu \mathrm{m}$ [6]. When bubbles reach the resonance size (note that in actual fact, resonance size refers to a range rather than a single size [7]), bubbles absorb the acoustic energy efficiently, grow to a maximum and undergo inertial collapse. The collapse of bubbles is near adiabatic that generates extreme temperatures and pressures transiently. Equations 3 and 4 could be used to estimate theoretical maximum temperature and pressure, respectively [4].

$$
T_{\max }=T_{0}\left\{\frac{\mathrm{P}_{\mathrm{m}}(\gamma-1)}{\mathrm{P}_{v}}\right\}
$$




$$
P_{\max }=P_{i}\left(\frac{P_{m}(\gamma-1)}{P_{i}}\right)^{\frac{\gamma}{(\gamma-1)}} \quad \text { Equation } 4
$$

$T_{\max }=$ maximum temperature; $T_{\mathrm{o}}=$ liquid temperature; $P_{\mathrm{m}}=$ liquid pressure, $P_{\mathrm{i}}=$ pressure inside the bubble, normally considered to be vapor pressure of the liquid $\left(P_{\mathrm{v}}\right) ; P_{\max }=$ maximum pressure; $\gamma=$ heat capacity ratio of the contents inside the bubble.

While theoretical estimates under specific conditions predict the temperatures and pressures to be of the order of more than $10000 \mathrm{~K}$ and 100's of atmospheres, respectively, experimentally determined temperatures are in the range 1000 - $5000 \mathrm{~K}$ [8,9]. Irrespective of these differences, such localised high temperatures, referred to as 'hot spots', are responsible for initiating a variety of redox reactions. In addition, bubble oscillations and collapse also generate a number of physical effects [1] that could be used to enhance rates of chemical processes and reactions due to enhanced mass transfer effects, as discussed in the following section.

\section{Sonochemistry}

Sonochemistry has a very broad meaning and could be described in many ways. In general, the term refers to sound-driven chemical reactions. Chemical reactions could be initiated by the acoustic cavitation process or may simply refer to increased reaction rates/efficiencies of reactions that would otherwise occur at a slower rate or lower efficiency.

First let us look at some examples where the reactions are initiated by acoustic cavitation process. In aqueous medium, water molecules could evaporate into the cavitation bubbles during the expansion phase of the acoustic cycle, which would experience the extreme heat generated on bubble collapse leading to the breaking of covalent bonds between $\mathrm{O}$ and $\mathrm{H}$ atoms. In argon saturated water, $\mathrm{OH}$ radicals and $\mathrm{H}$ atoms have been detected and quantified using several techniques [10-13].

$\left.\left.\left.\left.\left.\mathrm{H}_{2} \mathrm{O}\right)\right)\right)\right)\right) \mathbf{H}+\mathbf{O H} \quad$ (Reaction 1) (bold letters indicate radicals; “)))” refers to ultrasound)

For quantification, sophisticated techniques such as ESR were used. For example, Reisz and coworkers [10] used spin-traps to identify and quantify $\mathrm{H}$ and $\mathrm{OH}$, commonly referred to as primary radicals. Luminescence based techniques have also been used to quantify $\mathrm{OH}$ radicals. For example, reaction between terephthalic acid and $\mathrm{OH}$ radicals leads to the formation of hydroxyterephthalic acid, which has luminescent properties [11]. The reaction between $\mathrm{OH}$ radicals and luminol results in chemiluminescence (Figure 3) that could also be used for $\mathrm{OH}$ radical quantification [12]. 

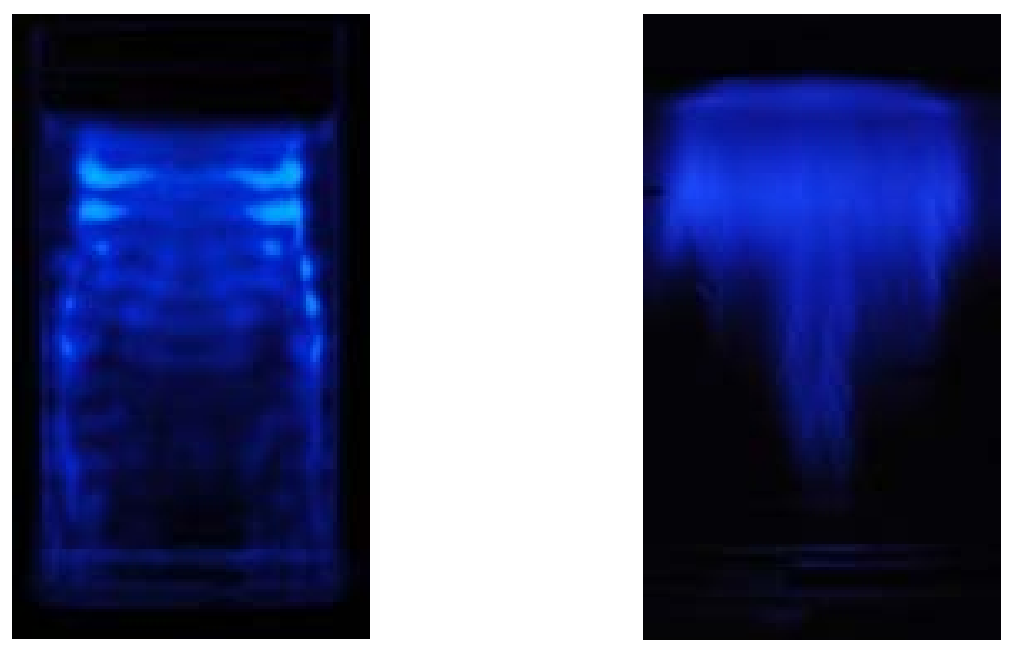

Figure 3: Sonochemiluminescence images observed at $440 \mathrm{kHz}$ and $700 \mathrm{kHz}$. The reaction between $\mathrm{OH}$ radicals and luminol in aqueous solution results in chemiluminescence, which could be used to visualise cavitation bubble field/structures in a reactor. The left image shows a clear standing wave pattern of antinodes (where active cavitation bubbles are located) and nodes. The right image does not show standing waves due to the domination of travelling waves that push active cavitation bubbles away from the source (transducer) located at the bottom [12].

A most common and absorption based technique is the iodide oxidation method (Weissler method) that quantifies $\mathrm{OH}$ radicals generated during acoustic cavitation by measuring the amount of $\mathrm{H}_{2} \mathrm{O}_{2}$ [13]. The oxidation of iodide to molecular iodine by $\mathrm{H}_{2} \mathrm{O}_{2}$ is the key reaction used in this quantification method, which are shown in Reactions 2 and 3.

$$
\begin{array}{cr}
2 \text { OH } \rightarrow \mathrm{H}_{2} \mathrm{O}_{2} & \text { Reaction (2) } \\
\mathrm{H}_{2} \mathrm{O}_{2}+2 \mathrm{I}^{-} \rightarrow \mathrm{I}_{2}+2 \mathrm{OH}^{-} & \text {Reaction (3) }
\end{array}
$$

Molecular iodine forms triiodide complex in aqueous solutions containing excess iodide that could be quantified spectrophotmetrically.

In air/oxygen saturated aqueous solutions, a number of other reactions (Reactions 4-8) could occur.

$$
\begin{array}{cr}
\mathrm{O}_{2} \rightarrow 2 \mathbf{O} & \text { Reaction (4) } \\
\mathbf{O}+\mathrm{O}_{2} \rightarrow \mathrm{O}_{3} & \text { Reaction (5) } \\
\mathbf{H}+\mathrm{O}_{2} \rightarrow \mathbf{H O}_{2} & \text { Reaction (6) } \\
\mathrm{N}_{2}+\mathrm{O}_{2} \rightarrow \mathrm{NO} & \text { Reaction (7) } \\
\mathrm{NO}+\mathrm{O}_{2} \rightarrow \mathrm{NO}_{2} & \text { Reaction (8) }
\end{array}
$$

What is interesting is that the primary radicals generated, namely, $\mathbf{H}$ and $\mathbf{O H}$, could be used for a variety of redox reactions. $\mathrm{H}$ atoms and $\mathrm{OH}$ radicals are strong reducing and oxidizing agents, respectively. From this view point, each of the acoustic cavitation bubble can be considered as an 
undivided electrochemical cell, where at the anode an oxidizing agent, and at the cathode a reductant is generated, and both species can react with constituents of the solution.

Let us consider a simple reduction reaction where $\mathrm{H}$ atoms could be used. One of the extensively studied sonochemical reactions is the formation of metal nanoparticles in aqueous solutions. For example, gold nanoparticles were sonochemically generated [14] in argon saturated aqueous solutions and a simplified reaction involves:

$$
\mathrm{AuCl}_{4}^{-}+3 \mathbf{H} \rightarrow \mathrm{Au}(0)+3 \mathrm{H}^{+}+4 \mathrm{Cl}^{-} \quad \text { (Reaction 9) }
$$

$\mathrm{Au}(0)$ agglomerates to generate Au nanoparticles.

Similarly, OH radicals could be used to oxidize organic pollutants [15] and simplified reaction involves:

$$
\text { Phenol }+\mathbf{O H} \rightarrow \text { dihydroxy phenols } \rightarrow \text { further oxidised products (Reaction 10) }
$$

It should also be considered that one may want to carry out only reduction or oxidation reactions. The question here is what do we do with other radicals? For example, if only reduction reaction is required, what do we do with $\mathrm{OH}$ radicals and if oxidation reaction is the main focus, how can we utilise $\mathrm{H}$ atoms? Here, the strategy generally is to follow what radiation chemists do, namely, convert the oxidizing radicals to reducing radicals for reduction focused reactions and vice versa.

Let us consider a reduction only reaction, such as Reaction (9). By adding a small amount of aliphatic alcohols (any organic in general would do this trick), $\mathrm{OH}$ radicals could be converted into alcohol radicals, which are strong reducing agents.

$$
\mathbf{H} / \mathbf{O H}+\mathrm{RCH}_{2} \mathrm{OH} \rightarrow \mathbf{R C H O H}+\mathrm{H}_{2} / \mathrm{H}_{2} \mathrm{O} \quad \text { (Reaction 11) }
$$

The alcohol radicals (RCHOH) are referred to as secondary radicals. Reaction (11) may occur within cavitation bubbles (since alcohol molecules are volatile and can diffuse into the bubble along with water molecules), at the bubble wall/solution interface (since alcohol molecules are surface active) and in the bulk solution ( $\mathbf{H} / \mathbf{O H}$ could diffuse out of the bubble into the bulk solution). It is also possible that evaporated alcohol molecules undergo pyrolysis under the extreme heat generated on bubble collapse and generate a variety of alkyl (R) radicals which are also strong reducing agents.

Hence, in this reducing environment, the reduction of gold chloride to gold nanoparticles would be dominated by the following simplified reaction (Reaction 12) [16].

$$
\mathrm{AuCl}_{4}^{-}+\mathbf{R C H O H} / \mathbf{R} \rightarrow \mathrm{Au}(0)+\text { other products } \quad \text { (Reaction 12) }
$$

Likewise, for an oxidation focused reaction, $\mathbf{H}$ atoms could be converted into an oxidising radical $\left(\mathbf{H O}_{2}\right)$ by Reaction 6 in oxygen/air saturated solutions. A number of examples where the redox radicals generated have been used will be discussed in the following sections. 
Acoustic cavitation could also be achieved in organic liquids. A variety of radical species were identified in chloroform and other organic solvents. For example, the sonication of chloroform generates a variety of radicals as shown in Reaction (13) [17].

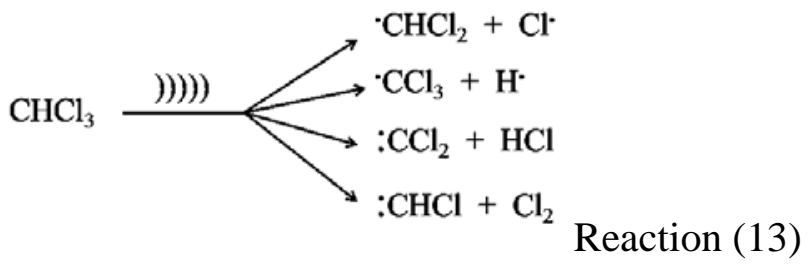

One other example that could be highlighted is the generation of amorphous nanomaterials in organic liquids [18]. When volatile metal complexes were dissolved in an organic solvent and sonicated, amorphous metal nanoparticles were formed. The reactions below show the formation of amorphous iron nanoparticles when octanol containing dissolved $\mathrm{Fe}(\mathrm{CO})_{5}$ was sonicated. The reason for the formation of amorphous metal nanoparticles is the fast cooling rates achieved during cavitation process. As discussed above, temperatures of a few thousand degrees are generated due to near adiabatic nature of bubble collapse. Volatile metal complexes diffuse into the cavitation bubbles during the expansion phase of acoustic cycle and decompose under the extreme heat conditions reached on bubble collapse. Since the hot spots are dispersed within the liquid that is at room temperature, the heat is quickly quenched by the surround liquid leading to cooling rates of $10^{9} \mathrm{~K} / \mathrm{s}$. Such fast cooling of the nanoparticles results in the formation of amorphous materials that show much higher catalytic activities compared those prepared conventionally [18].

The above mentioned examples highlight the chemical reactions induced by acoustic cavitation, that otherwise, would not occur or occur at much slower rates under normal conditions. The mass transfer effects generated due to acoustic cavitation could also be used to simply enhance the rates of chemical reactions or chemical processes such as emulsification (referred to as sonoprocessing). In particular, the mass transfer effects have been heavily used in heterogeneous systems [1-3].

Having introduced the basics of sonochemistry, the rest of the article will focus on applied sonochemistry with selected examples.

\section{Applied Sonochemistry}

The physical and chemical effects generated during acoustic cavitation have been used for a number of applications [1-3]. Processes such as emulsification, de-emulsification, extraction and cleaning are not covered here since they are classified under sonoprocessing. They do not refer to chemical reactions. However, in some sonochemical reactions such as emulsion polymerisation process, both the physical and chemical effects are required, which will be discussed below under polymers and biomedical applications. Another area where the physical effects of acoustic cavitation are 
increasingly being used is food/dairy processing. The physical and functional properties of dairy proteins, biopolymers, dairy fat, etc. have been modified by sonication process and extensive literature is available for the readers who may be interested in this area. Various chemical applications have been developed using the physical and chemical effects of acoustic cavitation, out of which three major areas are discussed below, namely materials synthesis, environmental and biomedical applications.

\subsection{Nanomaterials including polymers}

Synthesis of metal nanoparticles has been briefly discussed in Section 3 using gold as an example. Using a similar strategy, a variety of metal nanoparticles that include $\mathrm{Ag}, \mathrm{Cu}, \mathrm{Pt}$, $\mathrm{Rh}$, etc. have been sonochemically synthesised [19-25]. The choice of experimental conditions seem to affect the shape and size of nanoparticles generated. In general, smaller particles are generated when a higher amount of radicals are formed, which happened to be around $200 \mathrm{kHz}-600 \mathrm{kHz}$ [24]. A higher amount of radical production enhances the rate of nucleation resulting in smaller particles. In fact, the size of Au nanoparticles generated was shown to be controlled by the rate of radical production (see Figure 4). High Intensity Focused Ultrasound (HIFU) was used [25] to synthesis gold particles of different shapes as shown in Figure 4.
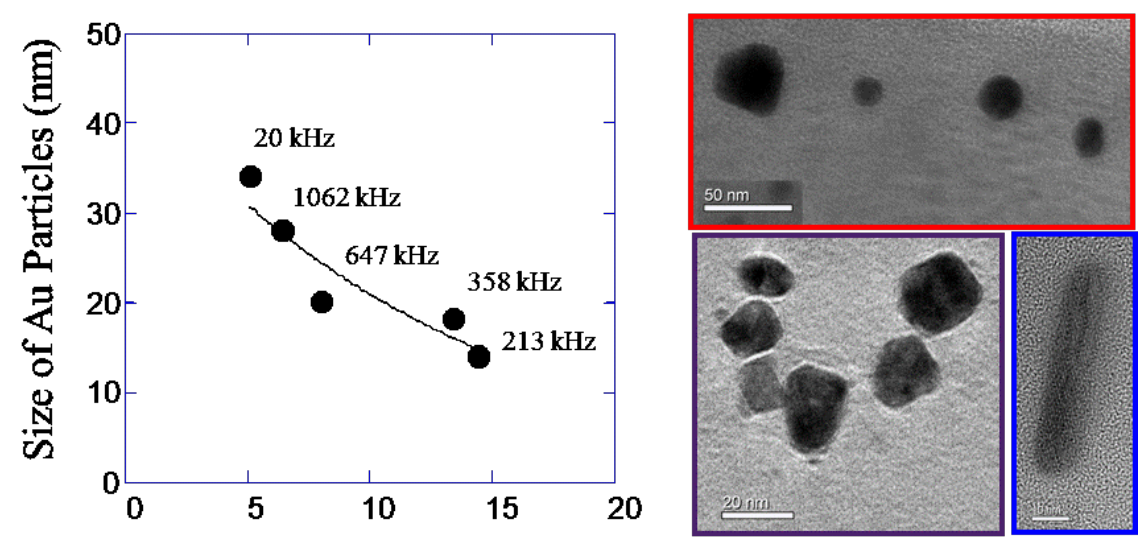

Rate of $\mathrm{Au}(\mathrm{III})$ reduction $\mu \mathrm{M} / \mathrm{min}$

Figure 4: The relationship between size of sonochemically generated gold nanoparticles as a function of rate of $\mathrm{Au}(\mathrm{III})$ reduction. It is reported that the rate of radical production is the lowest at $20 \mathrm{kHz}$ and follows the order $20 \mathrm{kHz}<1062 \mathrm{kHz}<647 \mathrm{kHz}<358 \mathrm{kHz}<213 \mathrm{kHz}$ [24]. The images of gold nanoparticles shown on the right show that the particle shape could be controlled, when HIFU is used under different experimental conditions [25].

Such metal particles have been found useful in many applications. For example, Au nanoparticles have been used in biomedical applications [1]. Metal particles deposited on other matrices such as graphene oxide layers have been used in catalytic and electrocatalytic applications. [1-3] 
Sonochemical synthesis of polymer latex particles [26-28] is another interesting application. A typical system involves an organic monomer and an aqueous system containing a stabiliser. Let us consider a specific example of synthesising poly methyl methacrylate (PMMA) latex particles. Methyl methacrylate monomer could be emulsified into an aqueous solution containing sodium dodecyl sulfate (SDS) as a stabiliser. The emulsification can be achieved by the shear forces generated at low $(20 \mathrm{kHz})$ frequency ultrasound. Such sono-emulsification process is very effective in generating small (about 50 - $200 \mathrm{~nm}$ in diameter) monomer droplets with a relatively narrow size distribution. The overall sono-emulsification and sono-polymerisation processes are depicted schematically in Figure 5.

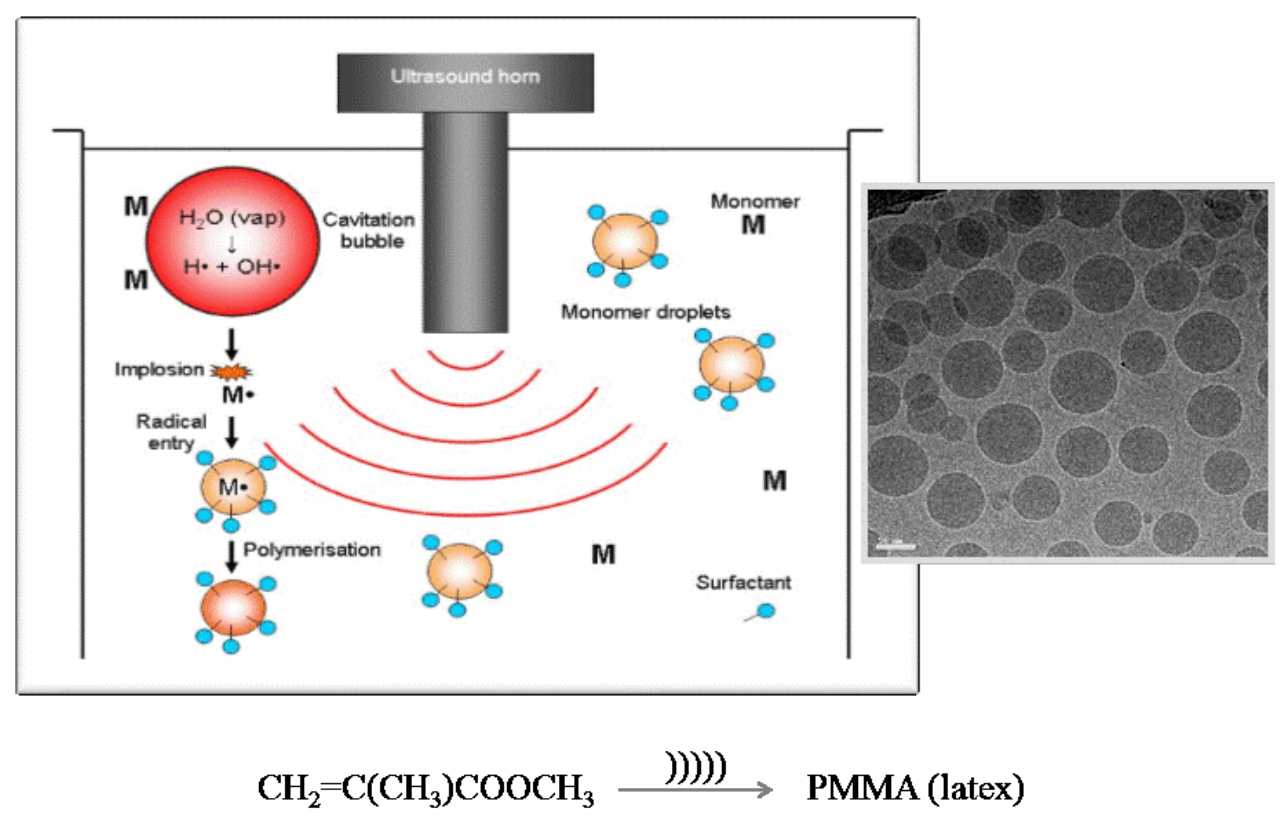

Figure 5: Schematic representation of ultrasonic emulsion polymerisation. The shear forces generated during acoustic cavitation emulsify the monomer in an aqueous medium. The primary and secondary radicals enter monomer droplets of about 50-100 nm and initiate polymerisation converting each droplet into a latex particle. The electron microscopic picture shows the latex particles (scale bar $=50 \mathrm{~nm}$ ). The reaction below shows the sonochemical polymerisation of methyl methacrylate to polymethylmethacrylate (PMMA). Adapted from Ref. [26-28].

Ultrasonic emulsion polymerisation has been used for synthesising magnetised polymer particles [28] that may have applications in biomedical imaging. In the synthetic process involved, magnetically active iron oxide nanoparticles were first dispersed in the monomer phase followed by emulsion polymerisation that resulted in the incorporation of iron oxide in the polymer latex matrix making the polymer particles magnetically responsive. In recent times, ultrasonic polymerisation is incorporated into RAFT [29] and ATRP [30] processes that resulted in polymers possessing “controlled” properties. 


\subsection{Environmental remediation}

The oxidative radicals generated during acoustic cavitation could be used to oxidise organic pollutants in an aqueous environment [31-34]. Taking benzoic acid as a simple organic pollutant, its sonochemical degradation is shown in Figure 6. Benzoic acid, being a weak acid exists in neutral or ionised form depending up on the solution $\mathrm{pH}$. The degradation rate vs concentration plots shown in Figure 6 suggests that the degradation efficiency is strongly dependent on the nature of the molecule. In its neutral form, benzoic acid is surface active and can adsorb at the bubble-solution interface. Due to the close proximity between the adsorbed molecules and $\mathrm{OH}$ radicals, generated within the bubbles, the rate of oxidation is higher leading to an efficient degradation. In its ionised form, benzoic acid is more hydrophilic and hence less prone to $\mathrm{OH}$ radical attack leading to a lower degradation rate. This is an important observation that suggests that the sonochemical degradation of organic pollutants strongly depends up on the surface activity of the pollutant molecules (further discussion is provided in Section 5. The mechanism of sonochemical degradation of organic pollutants involves the $\mathrm{OH}$ radical attack followed by completed mineralisation as shown in Figure 6.

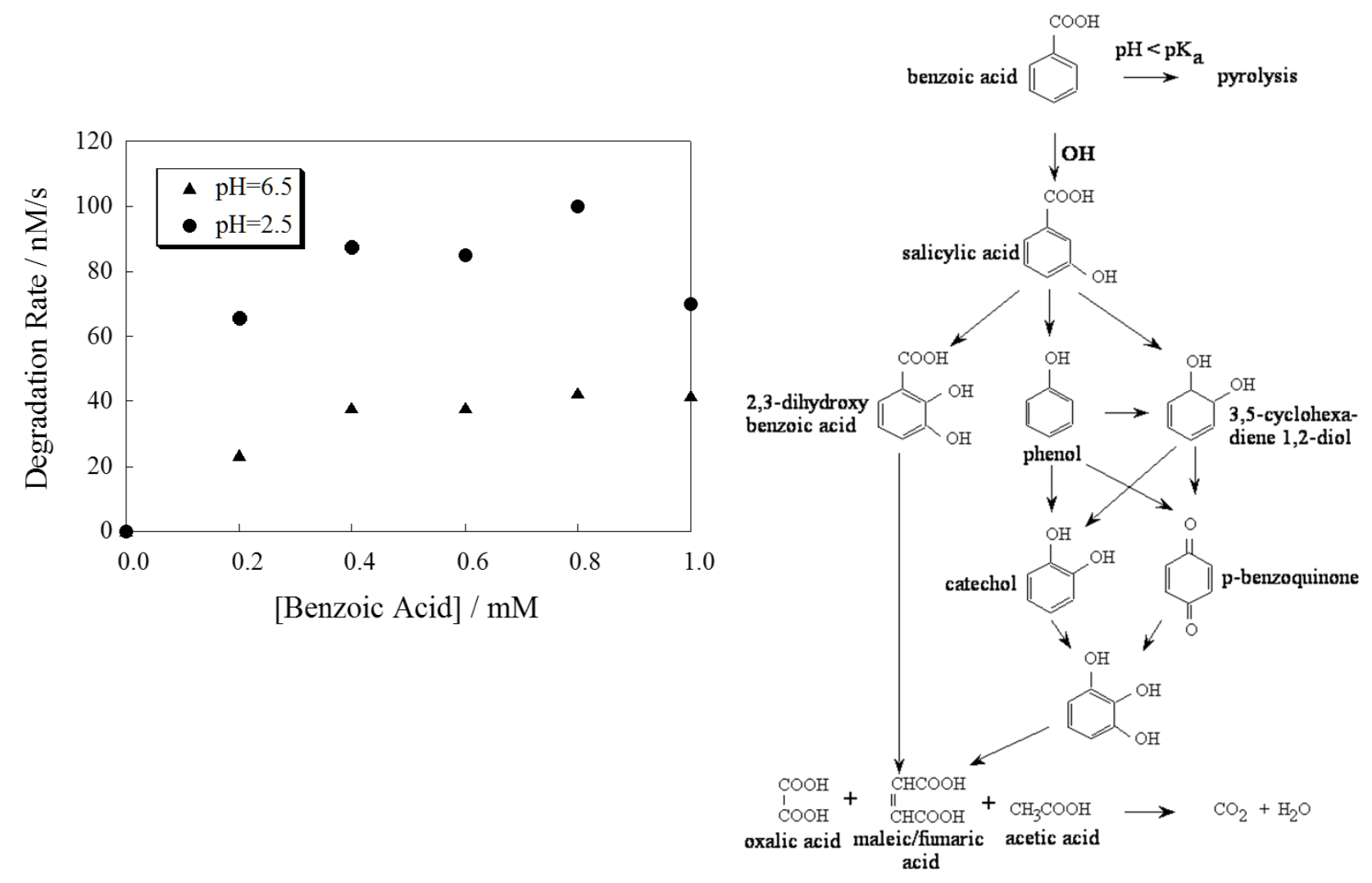

Figure 6: The dependence of sonochemical degradation rate of benzoic acid on solution $\mathrm{pH}$ and the degradation mechanism of benzoic acid [31]. 


\subsection{Biomedical applications}

Ultrasound is heavily used in imaging applications in diagnostic medicine [35]. In the past decade, ultrasonic synthesis of core-shell biomaterials has become an active research area [36-40]. Such materials could be used as ultrasound contrast agents or drug delivery vehicles. A variety biopolymers are used to make core-shell biofunctional materials, however, those biopolymers containing sulfhydryl functional groups are found to be stable. The formation of a core-shell structure involves the manipulation of the physical and functional properties of the biopolymer as well as the physical and chemical effects of cavitation. A strong physical shear is necessary to make an emulsion of air bubbles (for air-filled microspheres) or oil droplets (for drug filled microspheres) in the solution. The biopolymer needs to be surface active to stabilise the microbubbles or microdroplets. The oxidative radicals are used for cross-linking the adsorbed polymer molecules to generate a shell encapsulating air or oil/liquid drug.

Take a typical case of lysozyme-shelled air-filled microspheres [40]. Lysozyme is a globular protein and the structure is held together by intra-molecular disulfide bonds. In the synthetic process, Lysozyme is dissolved in water, partially denatured using a reducing agent to open up the structure in order to make the molecules surface active. The breaking of intramolecular disulfide bonds also generates free -SH groups. During the ultrasonic emulsification process, air bubbles are generated in the solution and lysozyme adsorbs at the surface of these air bubbles. During the cavitation bubble collapse, $\mathbf{H O}_{2}$ radicals are generated which oxidise - $\mathrm{SH}$ groups to form inter-molecular disulfide bonds creating a network of crosslinked proteins on the surface of the bubble leading to the formation of lysozyme-shelled microspheres (Figure 7). A similar procedure is followed to synthesise oil-filled microspheres. Both the core and shell could be functionalised for targeted drug delivery.

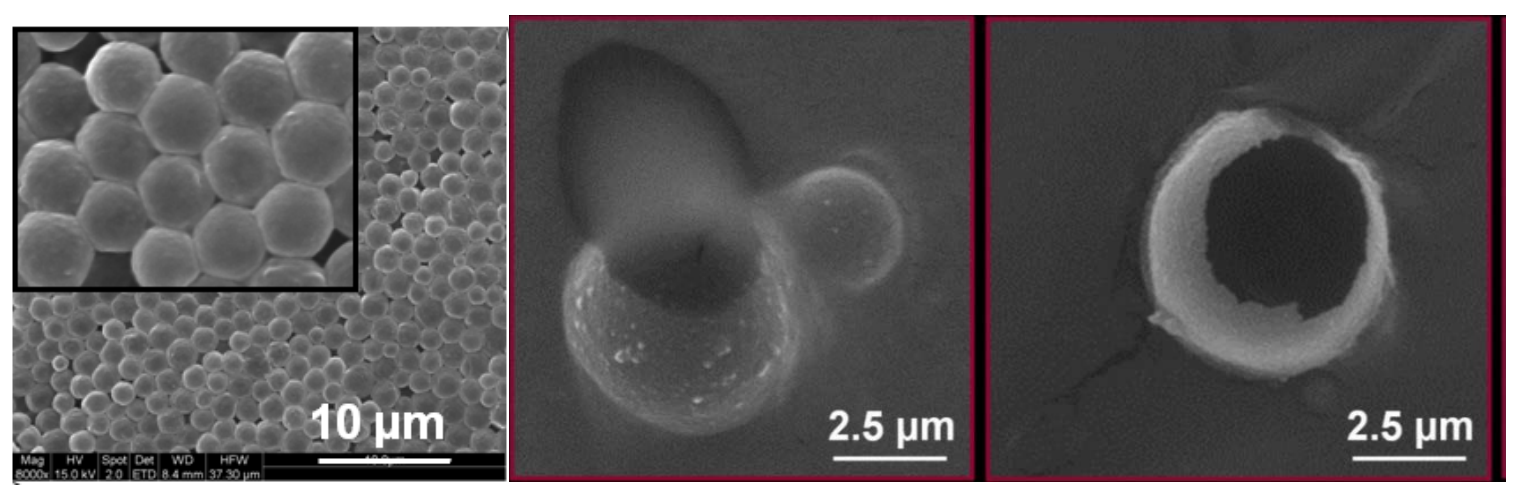

Figure 7: Electron microscopic images of ultrasonically synthesised lysozyme microspheres. Middle image shows the liquid core flowing out when the shell is broken and the right image shows the hollow core and thins shell. 


\section{Hybrid techniques}

One of the problems for the introduction of ultrasonics technology for large scale applications is the overall efficiency of the process vs cost of this technology. For some applications such as emulsification, extraction, etc., ultrasonic processing is found to be effective due to the very short sonication times required reducing the overall processing cost. However, for applications where the radicals are used, for example, in wastewater treatment, the overall efficiency is low due to the large amount of energy required to generate required amount of oxidising radicals for the mineralisation of organic pollutants. In the past 10 years or so, researchers have looked at combining two or more techniques to achieve synergistic advantages. One example of a hybrid technique is sonophotocatalytic process [41-42], a combination of sonolysis and photocatalysis, which is discussed in detail below. Other such hybrid techniques such as sonoelectrochemistry [43-44], sonoFenton [45], etc. have also been developed.

Sonochemical degradation of organic pollutants has selective advantages and disadvantages. This is demonstrated using benzoic acid as a model pollutant. Figure 6 shows the sonochemical degradation of benzoic acid as a function of solution pH. Benzoic acid is a weak acid with a pKa of about 4.2. Below pKa, benzoic acid exists in the neutral form and above pKa in its ionised form. In its neutral form, benzoic acid is relatively more hydrophobic and surface active whereas in the ionised form, it is less surface active due to hydrophilic characteristics.

Being surface active, the neutral form of benzoic acid adsorbs to cavitation bubble-solution interface and is prone to efficient $\mathrm{OH}$ radical (generated on bubble collapse) attack. Whereas the ionised form exists in the bulk solution, where $\mathrm{OH}$ radicals need to diffuse out of the bubble to react with benzoic acid, which is a relatively less efficient process due to very short lifetime of $\mathrm{OH}$ radicals. This is the reason for the observed difference in the degradation rates of benzoic acid at low and high $\mathrm{pH}$ conditions, observed in Figure 6.

What the above discussion suggests is that sonolysis is an efficient process for the degradation/oxidation of hydrophobic organic pollutants. Now, let us consider the mineralisation (complete degradation/oxidation of an organic compound to $\mathrm{H}_{2} \mathrm{O}$ and $\mathrm{CO}_{2}$ ) process under sonolytic conditions. TOC (Total Organic Carbon/Content) results for the sonochemical degradation of benzoic acid takes more than 24 hours for complete mineralisation of the parent compound and hence the process becomes energy intensive or less cost effective. The reason for such a long mineralisation process is the generation of hydrophilic intermediates due to the oxidation of parent compounds. See for example the nature of the products identified during the sonochemical degradation of benzoic acid shown in Figure 6. 
Photocatalysis [46] is a technique where light energy is used to generate redox species in semiconductors. In heterogeneous photocatalysis, UV/Visible light could be used to excite semiconductor particles to generate electrons and holes in conduction and valence bands, respectively. Most photocatalysts that have been extensively studied, for example $\mathrm{TiO}_{2}$, CdS, etc., have charges on the surface and hence efficiently degrade charged pollutants that are adsorbed on the surface of the photocatalytic particles. Hence, photocatalysis is an efficient process for the degradation of hydrophilic compounds. It can now be appreciated the reason for the hybridisation of the two oxidation techniques, sonolysis and photocatalysis - sonophotocatalysis. A few studies have shown synergistic enhancement [47] in the degradation and mineralisation rates of organic compounds in aqueous solutions.

\section{Instrumentation to generate acoustic cavitation}

In general, an ultrasonic reactor includes a wave generator, an amplifier and a transducer. Most commercial units put these components together in a single "cage”. For lab scale experiments, ultrasonic baths (ultrasonic cleaning baths are commonly available in the market) or transducers in the form of a horn (usually operates at $20 \mathrm{kHz}$ ) or a plate (usually operates above $100 \mathrm{kHz}$ ) are used. Ultrasonic baths operate at very low power density (since the volume of water used is significantly high) and hence generate very low amount of cavitation. $20 \mathrm{kHz}$ horn units operate at much high power densities since the energy is focussed at the tip of the horn. Plate-type transducers operate at moderate power densities. Further details on ultrasonic equipment are available in Ref. [1] and Ref. [48].

\section{Summary}

The interaction between bubbles in a liquid and ultrasound leads to acoustic cavitation under specific experimental conditions. Extreme conditions are generated in hotspots in solutions that are kept at room temperature. As a consequence of acoustic cavitation, highly reactive radicals and physical forces are generated that could be used in many chemical reactions and processes. A few examples of applied sonochemistry discussed in this article provide an indication of how ultrasound and sonochemistry could be used in multidisciplinary areas. Hybrid techniques utilising the advantages of acoustic cavitation have been developed. In recent years, dairy and food industries are investigating the benefits of ultrasonic processing as a non-thermal technology along with high pressure processing (HPP). Pharmaceutical industries on the other hand focus on the use of ultrasonics encapsulation technology for targeted drug delivery applications. In some countries like China, ultrasonic synthesis of graphene and other nanomaterials is tracking some attention. The basic and applied aspects discussed in this article may provide a good introduction to those interested in harvesting the benefits of ultrasonics and sonochemistry technology to develop various applications. 


\section{References}

[1] Ashokkumar M (Chief ed), Anandan S, Cavalieri F, Okitsu K, Yasui K, Chemat F. (Section eds), (2016) Handbook on Ultrasonics and Sonochemistry. Springer, Berlin

[2] Mason TJ (ed) (1990-2001) Advances in Sonochemistry Vol. 1-6. Elsevier, Amsterdam

[3] Mason TJ, Peters D (eds.) (2002) Practical Sonochemistry, Power ultrasound uses and applications. Ellis Horwood Publishers, Chichester

[4] Leighton TG (1994) The Acoustic Bubble. Academic Press, London.

[5] Crum LA (1980) J Acoust Soc Am 68:203-211

[6] Gaitan DF, Crum LA, Church CC, Roy RA (1992) J Acoust Soc Am 91:3166-3183

[7] Yasui K (2002) J Acoust Soc Am 112:1405-1413

[8] Misik V, Riesz P (1996) Ultrason Sonochem 3:25-37

[9] Hart EJ, Fischer CH, Henglein A (1990) Radiat Phys Chem, Int J Radiat Appl Instr Part C 36:511-516

[10] Misik V, Riesz P Ultrason Sonochem (1996) 3:S173-S186

[11] Price GJ, Lenz E (1993) Ultrasonics 31:451-456

[12] Ashokkumar M, Lee J, Iida Y, Yasui K, Kozuka T, Tuziuti T, Towata A (2009) PhysChemChemPhys 11:10118-10121

[13] Weissler A (1959) J Am Chem Soc 81:1077-1081

[14] Nagata Y, Mizukoshi Y, Okitsu K, Maeda Y. (1996) Radiat Res 146:333-338

[15] Berlan J, Tbelsi F, Delmas H, Wilhelm, AM, Petrrrignani JF (1994) Ultrason Sonochem 1:S97-S102

[16] Okitsu K, Teo BM, Ashokkumar M, Greiser F (2005) Aus J Chem 58:667-670

[17] Henglein A., Fischer C. H. Ber. Bunsen-Ges Phys Chem (1984) 88: 1196-1999

[18] Suslick KS, Hyeon T, Fang M, Cichowlas AA (1995) Mater Sci Eng A 204:186-192

[19] Nagata Y, Watananabe Y, Fujita SI., Dohmaru T, Taniguchi SJ (1992) Chem Soc, Chem Comm 1620-1622

[20] Okitsu K, Bandow H, Maeda Y (1996) Chem Mater 8:315-317

[21] Dhas NA, Raj CP, Gedanken NA (1998) Chem Mater 10:1446-1452

[22] Tanhaei M, Mahjoub AR, Safarifard V (2018) Ultrason Sonochem 41:189-195

[23] Khataee A, Saadi S, Vahid B, Joo SW (2016) J Ind Eng Chem 35:167-176

[24] Okitsu K, Ashokkumar M, Grieser F. (2005) J Phys Chem B 109:20673-20675

[25] Yusof NSM, Ashokkumar M (2015) Ultrason Sonochem 24:8-12

[26] Price GJ (2009) Polymer Int 58:290-295

[27] Teo BM, Grieser F, Ashokkumar M (2009) Macromol 42:4479-4483

[28] Teo BM, Suh SK, Hatton TA, Ashokkumar M, Grieser F Langmuir (2011) 27:30-33 
[29] McKenzie TG, Colombo E, Fu Q, Ashokkumar M, Qiao, GG (2017) Angewandte ChemieInt Ed 40:12302-12306

[30] Wang ZH, Wang ZH, Pan XC, Fu LY, Lathwal S, Olszewski M, Yan JJ, Enciso AE, Wang ZY, Xia HS (2018) Macro Lett 7:275-280

[31] Singla R, Ashokkumar M, Grieser F (2004) Res Chem Intermed 30:723-733

[32] Gokce TG, Nilsun IH (2011) Ultrason Sonochem 18:114-119

[33] Hamdaoui O, Merouani S (2017) Ultrason Sonochem 37:344-350

[34] Eslami A, Bahrami H, Asadi A. Alinejad A (2016) Water Sci Technol 73:1293-1300

[35] Nguyen FT, John R, Chaney EJ, Suslick KS, Boppart SA (2011) Cancer Res 71:4885, DOI: 10.1158/1538-7445.AM2011-4885

[36] Suslick KS, Grinstaff MW, Kolbeck KJ, Wong M (1994) Ultrason Sonochem 1:S65-S68

[37] Gedanken A (2008) Chem Eur J 14:3840-3853

[38] Wang X, Teng X (2010) Prog Chem 22:1086-1093

[39] Cavalieri F, Zhou M, Tortora M, Ashokkumar M (2012) Curr Pharmaceutical Design $18: 2135-2151$

[40] Cavalieri F, Ashokkumar M, Grieser F, Caruso F (2008) Langmuir 24:10078-10083

[41] Park JH (2013) Desal Wat Treatment 51:3106

[42] Babu SG, Ashokkumar M, Neppolian B (2016) Top Curr Chem 374:Article 75

[43] Gonzalez-Garcia J, Esclapez MD, Bonete P, Hernandez YV, Garreton LG, Saez V. (2010) Ultrason 50:318-322

[44] Durant A, Francois H, Reisse J, KirschDeMesmaeker A (1996) Electrochimica Acta 41:277284

[45] Harichandran G, Prasad S (2016) Ultrason Sonochem 29:178-185

[46] Marschall R, Wang L (2014) Catalysis Today 225:111-135

[47] Vinoth R, Karthik P, Devan K, Neppolian B, Ashokkumar M (2017) Ultrason Sonochem 35:655-663

[48] Ashokkumar M, Mason T (2017) Sonochemistry, Kirk-Othmer Encylcopedia of Chemical Technology, John Wiley \& Sons 


\section{University Library}

\section{- M M I N E R VA A gateway to Melbourne's research publications}

Minerva Access is the Institutional Repository of The University of Melbourne

Author/s:

Ashokkumar, M

Title:

Introductory text to sonochemistry

Date:

2018-06-01

Citation:

Ashokkumar, M. (2018). Introductory text to sonochemistry. CHEMTEXTS, 4 (2), https:// doi.org/10.1007/s40828-018-0061-4.

Persistent Link:

http://hdl.handle.net/11343/241897 